\title{
Teleoperation in Presence of Uncertainties: a Constraint-Based Approach
}

\author{
Gianni Borghesan, ${ }^{*}$ Bert Willaert, ${ }^{*}$ Tinne De Laet, ${ }^{*}$ \\ Joris De Schutter* \\ * Department of Mechanical Engineering, KU Leuven, \\ Celestijnenlaan 300B, B-3001 Leuven, Belgium. \\ (email: Gianni.Borghesan@mech.kuleuven.be)
}

\begin{abstract}
The application of teleoperation systems in the medical field demands for techniques that allow to add task constraints on top of (bilateral) teleoperation. How to blend these additional constraints into the control description of a teleoperation system remains a challenge. This paper shows how to realize a position-position and a position-force teleoperation scheme with additional task constraints using iTaSC. In particular, it shows how motion compensation, employing the estimate of such motion, can be achieved. As a case study, a periodical motion compensation for a simulated beating heart is implemented. The estimation of this periodical motion is based only on the position measurement of the end-effector of the slave robot, without using a camera or force sensor. Moreover, this paper presents an approach to gradually increase/decrease the motion compensation depending on the confidence in the estimated motion.
\end{abstract}

Keywords: Teleoperation, motion compensation, state estimation, medical application.

\section{INTRODUCTION}

The concepts of telepresence and teleoperation (where the main goal lies in obtaining a good transparency, regardless of delays in the communication channel) have been enriched with other concepts, where the control of the slave device is shared between the operator and an intelligent system. Some examples of such applications in the medical field are described by: Ren et al. (2008), who implement beating heart position compensation; Kragic et al. (2005) who implement virtual fixtures to guide surgeons during minimally invasive surgery procedures; and Kobayashi et al. (2007), who enforce tissue damage prevention for surgical applications. Common in these examples is that constraints have to be enforced on the pose of the robot or on the force that the robot applies. Moreover, these constraints are often easily expressed in local, and possibly moving, coordinates systems attached to specific objects (e.g. a patient organ/limb). For some cases the pose of these objects of interest are known or can be measured directly. In practice however, they often have to be estimated. The definition, as well as the implementation of the aforementioned constraints, benefits from a constraint-based programming approach like iTaSC (Instantaneous Task Specification using $C$ onstraints) proposed by De Schutter et al. (2007). The contributions of this framework are twofold: (1) it provides a systematic approach to obtain expressions for

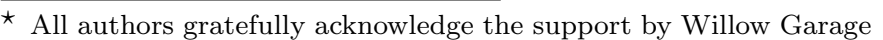
PR2 Beta program, KU Leuven's Concerted Research Action GOA/2010/011, KU LeuvenBOF PFV/10/002 Center-of-Excellence Optimization in Engineering (OPTEC), KUL BOF-IDO/09/012 Soft Tissue Overload Prevention, and European FP7 project RoboHow (FP7-ICT-288533). Tinne De Laet is a PostDoctoral Fellow of the Research Foundation - Flanders (FWO) in Belgium.
}

the task constraints and to evaluate these expressions at run time, and (2) it provides a systematic approach to model uncertainties in this geometric model and to estimate these uncertainties at run time. Software is available (Vanthienen et al., 2011) providing support to design and configure the different components within the $i T a S C$ -framework.

In previous work Borghesan et al. (2012) already demonstrated how iTaSC can be used to define a classical position-position scheme for a teleoperation system, while also allowing the integration of other constraints, in particular a virtual fixture. This paper progresses with respect to the state of the art in three aspects: i) In addition to the position-position scheme (first scenario), the force-position scheme (second scenario) is implemented in iTaSC. Both schemes are experimentally tested and compared; ii) While in previous work only simple constraints have been tested, here a motion compensation, using estimated motion, is described. More specifically, the scenario of a beating heart is investigated; and iii) An approach to gradually increase/decrease the motion compensation depending on the confidence in the estimated motion is presented. The particular case used in this paper involves a tele-operation task with the goal to operate on a moving heart (of which the motion is unknown) using a slave robot steered by an operator through the master robot. An overview of the experimental setup is shown in Fig. 2(b).

\section{ITASC MODELLING - GENERAL}

An iTaSC application consists of tasks, robots and objects, a scene-graph, and a solver. For every application, the programmer first has to identify the robots and objects. In the framework an object can be any object in the 


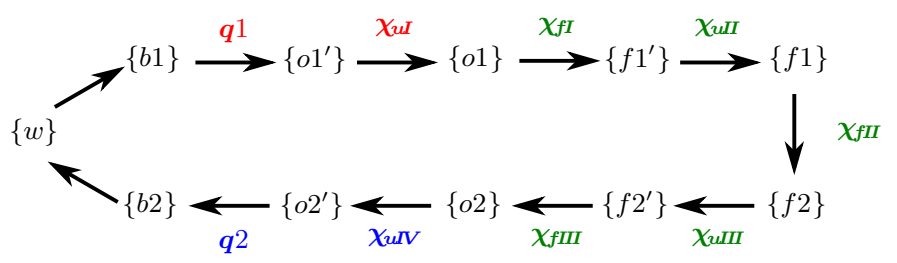

Fig. 1. Kinematic task loop with different frames and robot $(\boldsymbol{q})$, feature $\left(\boldsymbol{\chi}_{\boldsymbol{f}}\right)$, and uncertainty coordinates $\left(\chi_{u}\right)$. The primed frames represent the modelled frame poses while the others are the actual ones. $\boldsymbol{q} 1$ and $\boldsymbol{q} 2$ are the controllable DOF of the first and second object respectively (typically the robot joints). The feature coordinates $\chi_{f}$ are the DOF between $\{o 1\}$ and $\{o 2\}$, which, by introducing the feature frames, are distributed over three submotions: the relative motion of $\{f 1\}$ with respect to $\{o 1\}$ (submotion I, with feature coordinates $\left.\chi_{\boldsymbol{f I}}\right)$, the relative motion of $\{f 2\}$ with respect to $\{f 1\}$ (submotion II, with feature coordinates $\left.\chi_{\boldsymbol{f I I}}\right)$, and the relative motion of $\{o 2\}$ with respect to $\{f 2\}$ (submotion III, with feature coordinates $\left.\chi_{f I I I}\right)$ The uncertainty coordinates $\chi_{u}$ represent the pose uncertainty of the real object and feature frames with respect to the modelled ones: $\chi_{\boldsymbol{u}} \boldsymbol{I}$ represents the uncertainty of $\{o 1\}, \chi_{u}$ II represents the uncertainty of $\{f 1\}, \chi_{u} I I$ represents the uncertainty of $\{f 2\}$, and $\chi_{u \boldsymbol{I}} \boldsymbol{V}$ represents the uncertainty of $\{o 2\}$. The kinematic chain of $\{o 1\},\{o 2\}$, and the virtual kinematic chain (VKC) of the task are indicated in red, blue, and green, respectively.

robot system (for example the robot end-effector, link, or an object attached to it) or in the robot environment. Next, the kinematic chains of the robots and objects have to be defined as explained by De Schutter et al. (2007). These chains start at a reference frame (called base frame $\{b\}$ ) of the robot or object. The state of the kinematic chain is determined by the joint coordinates $\boldsymbol{q}$. Next, the programmer defines object frames $\{o\}$ on the robots and objects (i.e. frames on their kinematic chains) at locations where a task will take effect, for instance the robot endeffector or an object to be tracked.

The actual tasks define the space between pairs of object frames $(\{o 1\}$ and $\{o 2\})$, the feature space, as a virtual kinematic chain $(V K C)$. To simplify the task definition, feature frames are introduced (De Schutter et al., 2007). The feature frames are linked to an object, and indicate a physical entity on that object (such as a vertex or surface), or an abstract geometric property of a physical entity (such as the symmetry axis of a cylinder). Each task needs four frames: two object frames (called $\{o 1\}$ and $\{o 2\}$, each attached to one of the objects), and two feature frames (called $\{f 1\}$ and $\{f 2\}$, each attached to one of the corresponding features of the objects). For an application in 3D space, there are in general six DOF between $\{o 1\}$ and $\{o 2\}$. By introducing the feature frames, the six DOF are distributed over three submotions shown in Fig. 1: submotion I, between $\{f 1\}$ and $\{o 1\}$ (feature coordinates $\left.\chi_{\boldsymbol{f I}}\right)$, submotion $\mathbb{I}$, between $\{f 2\}$ and $\{f 1\}$ (feature coordinates $\chi_{\boldsymbol{f I I}}$ ), and submotion III, between $\{o 2\}$ and $\{f 2\}$ (feature coordinates $\chi_{f I I I}$ ).

Both the poses of the two objects and the poses of the two features with respect to the corresponding object can be uncertain. Uncertainty coordinates $\left(\boldsymbol{\chi}_{\boldsymbol{u} \boldsymbol{I}}, \boldsymbol{\chi}_{\boldsymbol{u} \boldsymbol{I I}}, \boldsymbol{\chi}_{\boldsymbol{u} \boldsymbol{I I I}} \&\right.$ $\left.\chi_{u} \boldsymbol{I} \boldsymbol{V}\right)$ are introduced to represent the pose uncertainty of a real frame with respect to a modelled frame (Fig. 1).

To obtain the desired task behaviour, one has to impose constraints on the relative motion between the two objects. To this end, the programmer has to choose the outputs that have to be constrained by defining an output equation: $\boldsymbol{y}=\boldsymbol{f}\left(\boldsymbol{q}, \boldsymbol{\chi}_{\boldsymbol{f}}\right)$. De Schutter et al. (2007) provide guidelines on how to define the task's VKC such that the outputs are simple functions, in most cases simple selectors, of the feature and joint coordinates. The imposed constraints used to specify the task are then directly expressed on the outputs as: $\boldsymbol{y}=\boldsymbol{y}_{d}$, for equality constraints, or $y \geqslant y_{d}$ or $y \leqslant y_{d}$, for inequality constraints. Each output constraint is enforced by a controller, which receives the desired output values $\left(\boldsymbol{y}_{d}\right)$ from a set-point generator.

Alternatively, the VKC introduced by a task can be used to define (virtual) measurements $\boldsymbol{z}$. To this end, the programmer has to define a measurement equation: $\boldsymbol{z}=\boldsymbol{h}\left(\boldsymbol{q}, \boldsymbol{\chi}_{\boldsymbol{f}}\right)$. Again, the task's VKC can be defined such that the measurements are simple functions, in most cases simple selectors, of the feature and joint coordinates. Remark that a measurement-related task does not introduce constraints, but rather helps to define (virtual) measurements easily using the feature coordinates of the task's VKC.

By defining the relations between the reference frames of the robots and objects and a global world reference frame $\{w\}$ in the scene-graph, the programmer defines how the robots and objects are located in the application scene. By connecting the VKC of the tasks to the object frames on the robots and objects, the programmer defines which robots execute the tasks on which objects. Each task defines a kinematic loop in the scene as shown in Fig. 1. The kinematic loops introduce constraints between the robot coordinates $\boldsymbol{q}$, the feature coordinates $\chi_{\boldsymbol{f}}=\left[\boldsymbol{\chi}_{\boldsymbol{f I}}{ }^{T}, \boldsymbol{\chi}_{\boldsymbol{f I I}}{ }^{T}, \boldsymbol{\chi}_{\boldsymbol{f I I I}}{ }^{T}\right]^{T}$, and uncertainty coordinates $\chi_{\boldsymbol{u}}=\left[\boldsymbol{\chi}_{\boldsymbol{u}}{ }^{T}, \boldsymbol{\chi}_{\boldsymbol{u} \boldsymbol{I I}}{ }^{T}, \boldsymbol{\chi}_{\boldsymbol{u} I I I}{ }^{T}, \boldsymbol{\chi}_{\boldsymbol{u} \boldsymbol{I}} \boldsymbol{V}^{T}\right]^{T}$, expressed by the loop closure equation: $\boldsymbol{l}\left(\boldsymbol{q}, \boldsymbol{\chi}_{\boldsymbol{f}}, \boldsymbol{\chi}_{\boldsymbol{u}}\right)=\mathbf{0}$.

The solver provides a solution for the optimization problem of calculating the desired robot joint values (i.e. the joint velocities $\dot{\boldsymbol{q}}_{d}$ for a velocity-based control scheme) out of the task constraints. This allows us to take into account different task priorities, different task constraint weights (in the case of conflicting constraints, i.e. overconstrained case), and weights for the joints of the robots (to solve the kinematic redundancy of the robot in the underconstrained case).

\section{ITASC MODELLING - TELEOPERATION APPLICATION}

This section details the iTaSC modelling of the teleoperation application. In particular, it discusses the different components of the iTaSC application: the robot and objects (Section 3.1), the tasks (Section 3.2), the scene-graph (Section 3.3), and the solver (Section 3.4). The general idea is to construct two separate iTaSC controllers: one for the simulated master and one for the simulated slave, that enforce different tasks defined to achieve the desired teleoperation behaviour. 


\subsection{Robots and objects}

The application involves two real robots (the master and the slave), two simulated robots (the master and the slave) (see the back-drivability task in Section 3.2), the patient's heart at the slave side, and the heart 'avatar' at the master side.

The real master and slave robots are simple serial three $\overline{\mathrm{DOF}}$ torque-controlled robots with joint angles $\boldsymbol{q}_{r}{ }^{1}$ . Object frames $\{o\}$ are defined on the real master and slave end-effectors. As the robots move in the application scene, their kinematic chains (and therefore also their end-effector poses) in the scene-graph are continuously updated using the robots' encoders.

The simulated master and slave robots have the same kinematic structure as the real master and slave robot, respectively and have joint angles $\boldsymbol{q}_{s}{ }_{1}$. In contrast to the real robots, the simulated robots act as pure integrators with respect to the commanded joint velocities $\left(\dot{\boldsymbol{q}}_{d}\right.$ calculated by the iTaSC controller):

$$
\boldsymbol{q}_{s}(t+\Delta t)=\boldsymbol{q}_{s}(t)+\dot{\boldsymbol{q}}_{d} \Delta t
$$

with $t$ the time and $\Delta t$ the time step. Object frames $\{o\}$ are defined on the simulated master and slave endeffectors.

The idea of using a real and simulated robot to obtain a back-drivable torque-controlled robot was introduced by Borghesan et al. (2012). The simulated robot, governed by the iTaSC controller, replaces the ideal velocitycontrolled robot of previous iTaSC implementations. In order to achieve the desired coupling between real and simulated robot, the real robot has track to the position of the simulated robot with a certain user-defined impedance. Therefore, the motor torques $\boldsymbol{\tau}$ are defined by the following proportional law:

$$
\boldsymbol{\tau}=\boldsymbol{K}_{\boldsymbol{q}_{r}}\left(\boldsymbol{q}_{s}-\boldsymbol{q}_{r}\right),
$$

where the gain matrix $\boldsymbol{K}_{\boldsymbol{q}_{\boldsymbol{r}}}$ is computed online in order to achieve a specific stiffness in the operational space described by $\boldsymbol{K}_{\boldsymbol{o}}{ }^{2}$ using:

$$
\boldsymbol{K}_{\boldsymbol{q}_{r}}=\boldsymbol{J}_{\boldsymbol{q} r}^{T} \boldsymbol{K}_{\boldsymbol{o}} \boldsymbol{J}_{\boldsymbol{q} r},
$$

with $\boldsymbol{J}_{\boldsymbol{q} r}$ the real robot Jacobian.

The patient's heart at the slave side, has an uncertain motion with respect to the world, modelled using uncertainty coordinates $\chi_{u}$. Section 4 elaborates on the motion model, estimator, and sensor measurements used to estimated the patient's heart motion. An object frame $\{o\}$ is attached to the location of interest on the heart and fixed with respect to the heart.

The avatar heart at the master side, is fixed with respect to the world, since this is the way the heart should be perceived by the operator at the master's side. The object frame $\{o\}$ is again attached to the location of interest on the avatar heart and fixed with respect to the avatar heart.

\footnotetext{
1 A superscript $m$ or $s l$ is added to indicate the master and slave robot whenever necessary: $\boldsymbol{q}_{r}^{m}$ and $\boldsymbol{q}_{r}^{s l}$ for the real master and slave, respectively and $\boldsymbol{q}_{s}^{m}$ and $\boldsymbol{q}_{s}^{s l}$ for the simulated master and slave, respectively.

2 Note that in Borghesan et al. (2012), a slightly different approach was followed, since the gain matrix $\boldsymbol{K}_{\boldsymbol{q}_{\boldsymbol{r}}}$ of the PR2 joint controller could not be changed online.
}

\subsection{Tasks}

As explained in Section 2, a task consists of the definition of a VKC between object frames (feature space), and in the case of output-related task: output equations, constraints on the outputs $\boldsymbol{y}$ of the task's kinematic loop, controllers enforcing the different constraints, and setpoint generators delivering the desired output values to the controllers; and in the case of a measurement-related task: measurement equations. This section specifies and details all the tasks involved in the application.

The teleoperation application involves different tasks. Whether the different tasks are active or not depends on the scenario (position-position or position-force teleoperation scheme).

The back-drivability task is specified in order to obtain a back-drivable robot, i.e. to make the simulated robot (interacting with iTaSC) react on the position of the real robot (and therefore of the forces exerted on it).

The real ee-position measurement task is specified in order to measure the position of the end-effector of the real slave robot with respect to the (estimated) real heart. In case of a position-position teleoperation scheme, there is an additional measurement task to measure the position of the end-effector of the real master robot with respect to the avatar heart.

In order to couple the position of the real master to the simulated slave, the position-coupling task couples the relative position of the real master end-effector (with respect to the avatar heart) to the relative position of the simulated slave end-effector (with respect to the real heart). In case of a position-position teleoperation scheme, there is not only position-coupling from the master to the slave but also vice versa, i.e. the relative position of the end-effector of the real slave (with respect to the real heart) is coupled to the relative position of the simulated master end-effector (with respect to the avatar heart).

In case of a position-force teleoperation scheme, there is an additional need for a force-coupling task reflecting the forces exerted on the slave end-effector to the master. The following paragraphs elaborate on the task definitions.

The back-drivability task defines a Cartesian feature space between the end-effector frame on the simulated robot $\left\{o 1^{b}\right\}$ and the end-effector frame on the real robot $\left\{o 2^{b}\right\}$ (this task can be defined for both the master real and simulated robot pair and the slave real and simulated robot pair).

The first feature frame $\left\{f 1^{b}\right\}$ coincides with $\left\{o 1^{b}\right\}$ and the second feature frame $\left\{f 2^{b}\right\}$ coincides with $\left\{o 2^{b}\right\}$. The feature coordinates defining the six DOF of the resulting $V K C$ are all located between $\left\{f 1^{b}\right\}$ and $\left\{f 2^{b}\right\}$, i.e. $\chi_{\boldsymbol{f I}}{ }^{b}=0$ and $\chi_{\boldsymbol{f I I}}{ }^{b}=0$, while an intuitive definition of $\chi_{\boldsymbol{f I I}}{ }^{b}$ is obtained by expressing these coordinates in the first feature frame $\left\{f 1^{b}\right\}: \chi_{\boldsymbol{f I}}{ }^{b}=\left[x^{b}, y^{b}, z^{b}, \alpha^{b}, \beta^{b}, \gamma^{b}\right]^{T}$, where $\left[x^{b}, y^{b}, z^{b}\right]^{T}$ define the $3 D$ position coordinates and $\left[\alpha^{b}, \beta^{b}, \gamma^{b}\right]^{T}$ define the $3 D$ orientation coordinates of the end-effector of the real robot with respect to the endeffector of the simulated robot.

The task constrains the three position DOF of the VKC in order to keep the position of the simulated robot close to the real robot. Therefore, the output vector $\boldsymbol{y}^{b}$ equals 


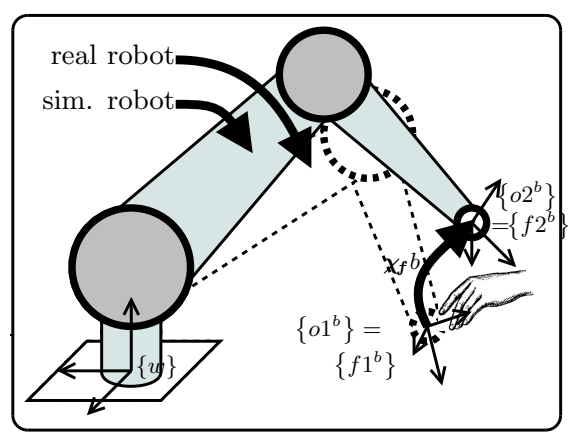

(a) Back-drivability constraint: the task coordinates are the position coordinates between the real and the simulated robot endeffectors.

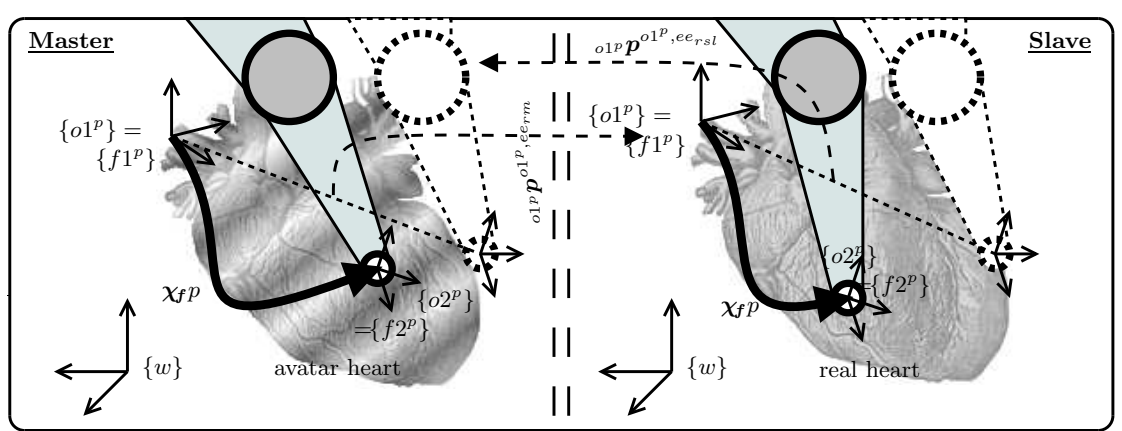

(b) Position-coupling constraint: the task coordinates are the position coordinates of simulated robots w.r.t. the real heart for the slave and with respect to the avatar heart for the master. The distances of the real (slave/master) robot end-effector to the (real/avator) heart are used as the desired outputs for the other (master/slave) position-coupling task.

Fig. 2. Figures (a) and (b) illustrate the frames and task coordinates for the back-drivability and position-coupling task respectively. Real robots are depicted with dashed lines contours, while the simulated robot are represented in solid contours.

the first three feature coordinates of vector $\chi_{f}{ }^{b}(1: 3)$. A proportional controller is used to achieve the desired fixed pose, which is delivered by a set-point generator simply generating a fixed value $\left(x_{d}^{b}=0 \mathrm{~m}, y_{d}^{b}=0 \mathrm{~m}, z_{d}^{b}=0 \mathrm{~m}\right)$. Fig. 2(a) shows the task coordinates of this task.

The real ee-position measurement task defines a Cartesian feature space between the heart (avatar heart for the master and real heart for the slave) $\left\{o 1^{r}\right\}$ and the end-effector frame on the real robot $\left\{o 2^{r}\right\}$.

The first feature frame $\left\{f 1^{r}\right\}$ coincides with $\left\{o 1^{r}\right\}$ and the second feature frame $\left\{f 2^{r}\right\}$ coincides with $\left\{o 2^{r}\right\}$. The feature coordinates defining the six DOF of the resulting $V K C$ are all located between $\left\{f 1^{r}\right\}$ and $\left\{f 2^{r}\right\}$, i.e. $\chi_{\boldsymbol{f I}}{ }^{r}=0$ and $\chi_{\boldsymbol{f I I I}}{ }^{r}=0$, while an intuitive definition of $\chi_{\boldsymbol{f I I}}{ }^{r}$ is obtained by expressing these coordinates in the first feature frame $\left\{f 1^{r}\right\}: \chi_{\boldsymbol{f I I}}^{r}=\left[x^{r}, y^{r}, z^{r}, \alpha^{r}, \beta^{r}, \gamma^{r}\right]^{T}$, where $\left[x^{r}, y^{r}, z^{r}\right]^{T}$ define the $3 D$ position coordinates and $\left[\alpha^{r}, \beta^{r}, \gamma^{r}\right]^{T}$ define the $3 D$ orientation coordinates of the end-effector of the real robot with respect to the endeffector of the simulated robot.

The measurement vector $\boldsymbol{z}^{r}$ equals the three position DOFs of the feature coordinate vector $\boldsymbol{\chi}_{\boldsymbol{f}}{ }^{r} . \boldsymbol{z}_{s l}^{r}$ indicates the position measurement of the real slave end-effector with respect to the real heart $\left({ }_{o 1^{r}} \boldsymbol{p}^{o 1^{r}, e e_{r}^{m}}\right)$, while $\boldsymbol{z}_{m}^{r}$ indicates the position measurement of the real master end-effector with respect to the avatar heart $\left({ }_{o 1^{r}} \boldsymbol{p}^{o 1^{r}, e e_{r}^{s l}}\right)$, where $e e_{r}^{m}$ and $e e_{r}^{s l}$ indicates the end-effector of the real master and real slave robot, respectively.

The position-coupling task defines a Cartesian feature space between the heart (avatar heart for the master and real heart for the slave) $\left\{o 1^{p}\right\}$ and the end-effector frame on the simulated robot $\left\{o 2^{p}\right\}$.

The first feature frame $\left\{f 1^{p}\right\}$ coincides with $\left\{o 1^{p}\right\}$ and the second feature frame $\left\{f 2^{p}\right\}$ coincides with $\left\{o 2^{p}\right\}$. The feature coordinates defining the six DOF of the resulting $V K C$ are all located between $\left\{f 1^{p}\right\}$ and $\left\{f 2^{p}\right\}$, i.e. $\chi_{\boldsymbol{f I}}{ }^{p}=0$ and $\chi_{\boldsymbol{f I I I}}{ }^{p}=0$, while an intuitive definition of $\chi_{\mathbf{f I I}}{ }^{p}$ is obtained by expressing these coordinates in the first feature frame $\left\{f 1^{p}\right\}: \chi_{\boldsymbol{f I I}}^{p}=\left[x^{p}, y^{p}, z^{p}, \alpha^{p}, \beta^{p}, \gamma^{p}\right]^{T}$, where $\left[x^{p}, y^{p}, z^{p}\right]^{T}$ define the $3 D$ position coordinates and $\left[\alpha^{r}, \beta^{r}, \gamma^{r}\right]^{T}$ define the $3 D$ orientation coordinates of the end-effector of the simulated robot with respect to the heart.

The task constrains the three position DOF of the VKC in order to control the relative position of the end-effector of the simulated master/slave robot with respect to the avatar/real heart close to the relative position of the endeffector of the real slave/master robot with respect to the real/avatar heart. Therefore, the output vector $\boldsymbol{y}^{p}$ equals the first three feature coordinates $\chi_{f}^{p}(1: 3)$. A proportional controller is used to achieve the desired pose, which is set equal to the relative position of the endeffector of the other (i.e. real master when defining the task for the slave and vice versa) real robot with respect to the (avatar/real) heart, i.e. $\left[x_{d}^{p}, y_{d}^{p}, z_{d}^{p}\right]^{T}=\boldsymbol{z}^{r}$.

Fig. 2(b) shows the task coordinates of this task.

The force-coupling task defines a Cartesian feature space between the end-effector frame on the simulated master robot $\left\{o 1^{f}\right\}$ and the end-effector frame on the real master robot $\left\{o 2^{f}\right\}$.

The first feature frame $\left\{f 1^{f}\right\}$ coincides with $\left\{o 1^{f}\right\}$ and the second feature frame $\left\{f 2^{f}\right\}$ coincides with $\left\{o 2^{f}\right\}$. The feature coordinates defining the three DOF of the resulting $V K C$ are all located between $\left\{f 1^{f}\right\}$ and $\left\{f 2^{f}\right\}$, i.e. $\chi_{\boldsymbol{f I}}{ }^{f}=0$ and $\chi_{\boldsymbol{f I I}}{ }^{f}=0$, while an intuitive definition of $\chi_{\boldsymbol{f I I}}{ }^{f}$ is obtained by expressing these coordinates in the first feature frame $\left\{f 1^{f}\right\}: \chi_{\boldsymbol{f I I}}{ }^{f}=\left[x^{f}, y^{f}, z^{f}\right]^{T}$, where $\left[x^{f}, y^{f}, z^{f}\right]^{T}$ define the $3 D$ position coordinates of the endeffector of the real robot master with respect to the endeffector of the simulated master robot.

The task constrains all three position DOF of the VKC in order to reflect all forces from the slave side to the master side. Therefore, the output vector $\boldsymbol{y}^{f}$ equals the three position feature coordinates $\chi_{f}^{f}(1: 3)$. A proportional controller is used to control this output to a desired pose that is calculated from the real slave end-effector forces $\boldsymbol{f}^{e e_{r}^{s l}}$ using the operational space stiffness $\boldsymbol{K}_{\boldsymbol{o}}$, i.e. $\boldsymbol{y}_{d}^{f}=\boldsymbol{K}_{\boldsymbol{o}}{ }^{-1} \boldsymbol{f}^{e e_{r} s l}$. 

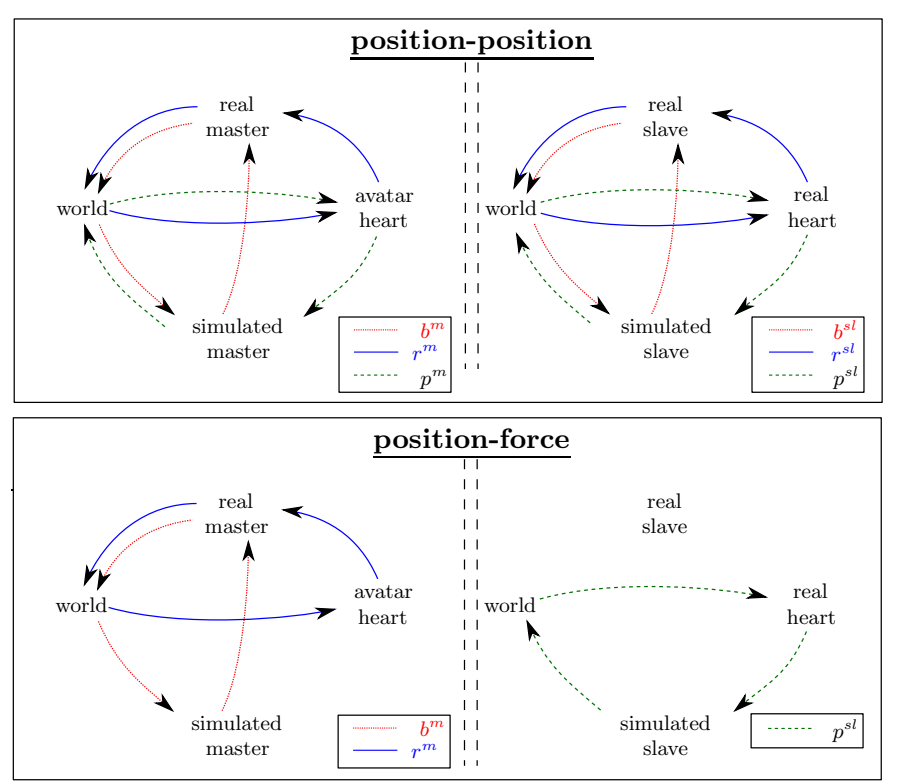

Fig. 3. Overview of the different loops in the scene-graph.

Table 1 gives an overview of which output-related tasks are active for the two scenarios (position-position or positionforce scheme) and details the set-points $\boldsymbol{y}_{d}$ for the task constraints.

\begin{tabular}{c|ccc:cc}
$\boldsymbol{y}_{d}$ & $b^{m}$ & $p^{m}$ & $f^{m}$ & $b^{s l}$ & $p^{s l}$ \\
\hline Pos-Pos & $\boldsymbol{O}$ & $\boldsymbol{z}_{s l}^{r}$ & $\mathrm{x}$ & $\boldsymbol{O}$ & $\boldsymbol{z}_{m}^{r}$ \\
Pos-For & $\mathrm{x}$ & $\mathrm{x}$ & $\boldsymbol{K}_{\boldsymbol{o}}{ }^{-1} \boldsymbol{f}^{e e_{r}^{s l}}$ & $\mathrm{x}$ & $\boldsymbol{z}_{m}^{r}$ \\
\hline
\end{tabular}

Table 1. Overview of which output-related tasks are active for the two scenarios (positionposition or position-force scheme) and setpoints $\boldsymbol{y}_{d}$ for the task constraints (' $\mathrm{x}$ ' indicates an inactive task). $b^{m}$ and $b^{s l}$ indicate the back-drivability task for the master and slave, respectively. Likewise $p^{m}$ and $p^{s l}$ indicate the position-coupling task for the master and slave, respectively. $f^{m}$ indicates the force-coupling task, which is defined for the simulated master.

\subsection{The scene-graph}

The scene-graph (represented in Fig. 3) keeps track of all robots and objects involved in the application, and in particular of their poses with respect to the world reference frame $\{w\}$, and indicates which robots execute the tasks on which objects. From the scene graph it can be seen that since the real ee-position measurement task and the position-coupling tasks for the slave are closed over the real heart, the feature coordinates of these tasks will depend on the estimated uncertainty coordinates describing the motion of the real heart.

\subsection{The solvers}

Two separate iTaSC solvers are constructed, one to calculate the desired joint velocities $\dot{\boldsymbol{q}}_{d, s}^{m}$ of the simulated master, and the other to calculate the desired joint velocities $\dot{\boldsymbol{q}}_{d, s}^{s l}$ of the simulated slave, employed in place of $\dot{\boldsymbol{q}}_{d}$ in Eq. (1). Both solvers solve an optimization problem involving the task constraints, the constraint weights, the task priorities, the estimated uncertainty coordinates, and the robot weight matrices. In this application the solver has to calculate the desired velocities for i) the three controllable DOF (each) of the simulated master and slave robots out of six constraints (each) in the positionposition teleoperation scheme, thus leading to an overconstrained problem, ii) the three controllable DOF (each) of the simulated master and robots out of three constraints (each) in the position-force teleoperation scheme. Equal task priorities and equal robot joint weights are used. Table 2 gives an overview of the number of constraints for the two scenarios for both the simulated master and simulated slave robot.

\begin{tabular}{c|ccc:cc}
$\sharp$ constraints & $b^{m}$ & $p^{m}$ & $f^{m}$ & $b^{s l}$ & $p^{s l}$ \\
\hline Pos-Pos & 3 & 3 & 0 & 3 & 3 \\
Pos-For & 0 & 0 & 3 & 0 & 3 \\
\hline
\end{tabular}

Table 2. Overview of the number of constraints introduced by each output-related task for the two scenarios (position-position or positionforce scheme) $b^{m}$ and $b^{s l}$ indicate the backdrivability tasks ( imposed master/slave), $p^{m}$ and $p^{s l}$ indicate the position-coupling task (master/slave), and $f^{m}$ indicates the forcecoupling task ( defined only for master).

\section{HEART MOTION}

To be able to compensate for the movement of the heart, the motion has to be estimated. The motion of the heart is observed using the end-effector position of the real slave robot only while the slave robot is in contact with the real heart. This section first explains how the unknown heart motion can be modelled and estimated using the endeffector position measurements of the slave robot while in contact with the real heart. Next, it is shown how the estimated heart motion can be used as a feed-forward signal in the control.

\subsection{Heart motion modelling}

For sake of simplicity, this paper assumes a sinusoidal heart motion along a known direction, in this case the $y$-direction of the world frame $\{w\}$. Following the iTaSC framework, the uncertain motion of the heart is modelled using uncertainty coordinates $\chi_{u}\left(\chi_{u} \boldsymbol{I}\right.$ for the positioncoupling task of the simulated slave $\left(p^{s l}\right)$, and for the real ee-position measurement task of the simulated slave $\left.\left(r^{s l}\right)\right)$. In this case we need only one uncertainty coordinate representing the uncertain $y$-position of the real heart i.e.:

$$
\boldsymbol{T}_{w}^{o 1_{r}^{p}}\left(\boldsymbol{\chi}_{\boldsymbol{u}}\right)=\left[\begin{array}{c|c} 
& 0 \\
\boldsymbol{I}_{3 \times 3} & \chi_{\boldsymbol{u}} \\
& 0 \\
\hline \boldsymbol{O}_{1 \times 3} & 1
\end{array}\right], \quad{ }_{w}^{o 1^{p}} \mathbf{t}_{w}^{o 1^{p}}\left(\dot{\boldsymbol{\chi}}_{\boldsymbol{u}}\right)=\left[\begin{array}{c}
0 \\
\dot{\chi}_{\boldsymbol{u}} \\
\boldsymbol{O}_{4 \times 1}
\end{array}\right],
$$

where ${ }_{w}^{o 1^{p}} \mathbf{t}_{w}^{o 1^{p}}$ is the twist of the heart with respect to the world expressed in the world and with velocity reference point the origin of $\left\{o 1^{p}\right\}$.

Since the heart's motion is sinusoidal, $\chi_{\boldsymbol{u}}$ can be modelled as:

$$
\chi_{\boldsymbol{u}}=x_{o f f}+A \sin (\theta),
$$

with $x_{\text {off }}$ the offset, $A$ the unknown amplitude, and $\theta=$ $\omega t+\phi$, with $\omega$ the unknown frequency and $\phi$ the unknown 
phase of the sinusoidal motion. Therefore, instead of directly estimating $\chi_{\boldsymbol{u}}$, we estimate $\boldsymbol{x}=\left[x_{o f f}, A, \omega, \theta\right]$. Following Yuen et al. (2009), the process model is:

$$
x[k+1]=\left[\begin{array}{cccc}
1 & 0 & 0 & 0 \\
0 & 1 & 0 & 0 \\
0 & 0 & 1 & 0 \\
0 & 0 & \Delta t & 1
\end{array}\right] x[k] .
$$

As mentioned earlier, the heart motion is measured using the $y$-position of the real slave end-effector ${ }_{w} \boldsymbol{p}^{w, e e_{r s l}}$ while in contact. The measurement model of the system and the measurement Jacobian $H$ become:

$$
\begin{aligned}
& {\left[\begin{array}{lll}
0 & 1 & 0
\end{array}{ }_{w} \boldsymbol{p}^{w, e e_{r s l}}=x_{o f f}+A \sin (\theta)\right. \text { and }}
\end{aligned}
$$

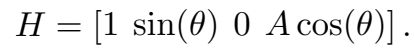

\subsection{Heart motion estimation}

In this paper we use an Extended Kalman filter to estimate the heart motion. The EKF involves a prediction step and a correction step. The estimation is updated via the prediction at each sample time, while the correction is executed only when a useful measurement is acquired. The endeffector position measurement only provides information about the heart's motion if the end-effector is in contact with the environment. A measurement is considered useful when it can be explained by the process and measurement model and the current estimate (indicated by the Normalised Innovation Squared (NIS) test, see Lefebvre et al. (2003), checking whether the innovation is consistent with the model with a likeness of $70 \%$.) and when the interaction force between the robot and the environment is above a certain threshold, i.e. the robot is considered to be 'in contact'. To monitor this, it is continuously checked whether the error between the desired and the actual slave robot position exceeds a threshold of $2 \mathrm{~mm}$. Taking into account the stiffness of the position controller of the robot, i.e. $1000 \mathrm{~N} / \mathrm{m}$, this corresponds to a threshold at $\approx 2 \mathrm{~N}$. From the estimated state $\hat{\boldsymbol{x}}=\left[\widehat{x_{o f f}}, \hat{A}, \hat{\omega}, \hat{\theta}\right]$, the estimated uncertainty coordinate and estimated uncertainty coordinate time derivatives are calculated as:

$$
\begin{aligned}
& \widehat{\chi}_{\boldsymbol{u}}=\hat{A} \sin (\hat{\theta}) \text { and } \\
& \widehat{\dot{\chi}}_{\boldsymbol{u}}=\hat{A} \hat{\omega} \cos (\hat{\theta}) .
\end{aligned}
$$

In the above equation, $\widehat{x_{o f f}}$ is disregarded in the computation of $\widehat{\chi}_{\boldsymbol{u}}$, since only the the periodical motion and not for the absolute position of the heart has to be compensated. This solution is adopted since measurements take place only when the slave robot achieves contact with the heart, and compensating for $\widehat{x_{o f f}}$ would cause the slave to index its position (while exerting a force on the heart). In case the position of the heart is known, $\left\{o 1^{p}\right\}$ can be placed in such a way that $x_{o f f} \approx 0$, i.e. $\left\{o 1^{p}\right\}$ is near the contact point. In this case $\widehat{x_{o f f}}$ can be reintroduced, allowing for the estimation and compensation of the periodical movements and its absolute position. Note that absolute position compensation is not strictly necessary in a teleoperation system, since the operator takes care of it.

\subsection{Heart motion in control}

This section details the two ways the estimated heart motion is used in iTaSC to control the simulated slave.
First of all, the estimated heart motion is used to update the position of the real heart with respect to the world $\boldsymbol{T}_{w}^{o 1_{r}^{p}}\left(\chi_{\boldsymbol{u}}\right)$ as explained by (4). Secondly, the solver also takes into account the estimated uncertainty coordinates when calculating the desired joint velocities $\dot{\boldsymbol{q}}_{d, s}^{s l}$ of the slave as explained in Section 3.4. In particular, $\dot{\boldsymbol{q}}_{d}$ are calculated as follows:

$$
\begin{gathered}
\dot{\boldsymbol{q}}_{d, s}^{s l}=\boldsymbol{A}_{\boldsymbol{W}}^{\dagger}\left(\dot{\boldsymbol{y}}+\boldsymbol{B} \widehat{\dot{\chi}}_{\boldsymbol{u}}\right), \text { with } \\
\boldsymbol{A}=\boldsymbol{C}_{\boldsymbol{f}} \boldsymbol{J}_{\boldsymbol{f}}^{-1} \boldsymbol{J}_{\boldsymbol{r}}, \quad \boldsymbol{B}=\boldsymbol{C}_{\boldsymbol{f}} \boldsymbol{J}_{\boldsymbol{f}}^{-1} \boldsymbol{J}_{\boldsymbol{u}},
\end{gathered}
$$

where: $\boldsymbol{J}_{\boldsymbol{r}}, \boldsymbol{J}_{\boldsymbol{f}}$, and $\boldsymbol{J}_{\boldsymbol{u}}$ are the Jacobian matrices that map the time-derivative of the joint, feature, and uncertainty coordinates into a twist expressed in a world frame, and $\cdot_{W}^{\dagger}$ is a weighted pseudo-inverse taking into account the constraint weights, task priorities, and robot weight matrix.

In the presented teleoperation application however, the uncertainty on the estimated heart motion can be high if no useful measurements have been available for a certain time (see Section 4.2) or the EKF estimate can not have converged yet or be unstable. In these cases, the confidence in the estimated heart motion is low and it is not desirable to use the estimated $\widehat{\chi}_{\boldsymbol{u}}$ to update $\boldsymbol{T}_{w}^{o 1_{r}^{p}}\left(\widehat{\chi}_{\boldsymbol{u}}\right)$ nor to use $\hat{\dot{\chi}}_{\boldsymbol{u}}$ as a feedforward signal. Therefore, this paper proposes to use a confidence index $i_{r} \in[0,1]$ to activate/deactivate the estimated uncertainty within iTaSC depending on the confidence in the estimated uncertainty as:

$$
\boldsymbol{T}_{w}^{o{ }_{r}^{p}}\left(i_{r} \boldsymbol{\chi}_{\boldsymbol{u}}\right) \quad \text { and } \quad \dot{\boldsymbol{q}}_{d, s}^{s l}=\boldsymbol{A}_{\boldsymbol{W}}^{\dagger}\left(\dot{\boldsymbol{y}}+i_{r} \boldsymbol{B} \widehat{\dot{\boldsymbol{\chi}}}_{\boldsymbol{u}}\right)
$$

for the estimated heart position and the estimated heart velocity feedforward, respectively. Since the changes in the heart position and feedforward signals should be smooth, the confidence index is only gradually adapted as:

$i_{r}[k]= \begin{cases}i_{r}[k-1]+\Delta i_{r} & \text { if 'in contact' \& 'EKF stable' } \\ i_{r}[k-1]-\Delta i_{r} & \text { if 'in contact' \& 'NIS test fails' } \\ i_{r}[k-1] & \text { otherwise, }\end{cases}$

where $\Delta i_{r}$ is the allowed incremental change of $i_{r}$ during one time step. Section 4.2 already gave the details of the 'in contact' and 'NIS test fails' conditions. The condition 'EKF stable state' is assumed to hold when the estimated amplitude $\hat{A}$ is inside a certain boundary for a specific time window, i.e. if it behaves as described by (6). Note that this ad hoc solution should be substituted by a more general approach in future works.

\section{EXPERIMENTS}

The two scenarios (position-position and position-force scheme) are tested in a teleoperation setup that consists of two three DOF robotic devices with the same serialparallel kinematic structure as the PHANTOM Premium haptic device (Massie and Salisbury, 1994). Those two robots are designed and built at the KU Leuven (Willaert et al., 2012). In comparison with the PHANTOM, the robots have a 10 times higher output force, the structural stiffness is improved by a factor of four and, due to the use of a gearbox (1:5.8) and a high resolution encoder, the gains for position and velocity loops can be a factor five higher. The beating 'heart' is realized by a one DOF device with a current-driven voice-coil motor. The master and slave robots have a force sensor mounted on the endeffector, and the beating heart is equipped with a rubber 

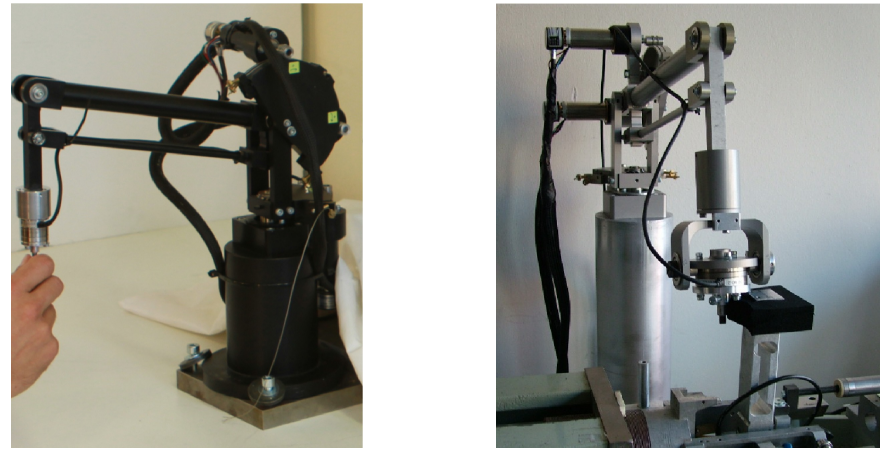

Fig. 4. Picture of the master robot (left), and slave robot and the 'heart' device (right).

ring that protects the slave sensor. The system is shown in Fig. 4.

Each scheme is tested with an experiment that consists of three phases:

(1) the slave robot, initially not in contact with the heart, is moved towards it (from $0 \mathrm{~s}$ to $c a .3 \mathrm{~s}$ for positionposition and position-force),

(2) the user enforces the contact between the slave and the heart until the EKF reaches a steady state and the estimated heart motion is being used in the control $\left(i_{r}>0\right)$. At this point the user starts to experience the compensation (from $3 \mathrm{~s}$ to $c a$. $12.5 \mathrm{~s}$ for positionposition and from $c a .3 \mathrm{~s}$ to $c a .14 .5 \mathrm{~s}$ for positionforce),

(3) when the user releases contact, the slave continues to compensate for the estimated motion, following the model expressed in (6) (from $c a$. 12.5 s to the end for position-position and from $c a .14 .5 \mathrm{~s}$ to the end for position-force).

The results for the two scenarios are shown by a number of figures: Fig. 5(a), Fig. 6(a), and Fig. 7(a) refer to the experiments with the position-position scheme, while Fig. 5(b), Fig. 6(b), and Fig. 7(b) refer to the experiments with the position-force scheme. Fig. 5 shows from top to bottom: the $y$ coordinate of the end-effector positions of master and slave expressed in their respective robot bases, the same positions expressed in respectively the 'avatar' and the 'heart' frame $\left(\left\{o 1^{p}\right\}\right)$, the $y$ component of measured forces, expressed in their respective robot bases, and the confidence index $i_{r}$, along with the binary information that expresses whether the slave is 'in contact'. Fig. 6 reports the state of the EKF, in solid lines. The dashed lines show the 'nominal' state, i.e. the parameters used to command the sinusoidal motion of the 1-DOF device. The first parameter, the $x_{o f f}$, is considered unknown a priori, since it depends on the initial position of the 1-DOF setup w.r.t. the slave robot. Fig. 7, finally, reports the measured (dashed line) position and velocity of the heart, along with the $y$-axis motion and velocity (solid lines).

As could be expected, the transparency of the positionforce scheme is better than the other scheme, since in the former the dynamics of the slave are not affecting the reflected force. Obviously, this advantage comes at the cost of force sensing. The stiffness is similar for both scheme and is defined a.o. by $\boldsymbol{K}_{\boldsymbol{o}}$, which is chosen to be a diagonal matrix with $1000 \mathrm{~N} / \mathrm{m}$ as diagonal elements.
The three phases described above can be distinguished in the figures: before the first contact, $i_{r}=0$ and therefore no compensation is active. During the first seconds in which the slave makes contact with the heart, both the master and the slave follow the sinusoidal motion. After the EFK reaches the equilibrium, $i_{r}$ goes from 0 to 1 and the compensation becomes active. This compensation remains active after loosing contact (as shown in the bottom graphs of Fig. 5). In future work, we will additionally investigate how to decrease the $i_{r}$ after long long of contact by for instance making the $i_{r}$ depend on the uncertainty of the estimated heart motion. The effect of the compensation can be appreciated by comparing the first and the second graph of Fig. 5: in the second phase of both experiments, the master robot stops to follow the sinusoidal motion of the heart, while the slave robot continues, in the absolute world frame $\{w\}$, but is standing still with respect to the estimated real heart $\left(\left\{o 1^{p}\right\}\right)$, and reflecting the master position w.r.t. $\left\{o 1^{p}\right\}$. This shows that the operator does not have to compensate for the sinusoidal motion of the heart as soon the confidence in the heart motion estimation is high enough. The activation of the motion compensation is also clearly visible by the solid line in Fig. 7 .

\section{CONCLUSION}

This paper demonstrated the iTaSC approach to a teleoperation task for both the position-position and positionforce teleoperation scheme. In particular, it showed how motion compensation can be achieved using the example of a beating heart. The motion compensation can be gradually increased and decreased depending on the confidence in the estimated motion.

In doing so, a system that is able to compensate for a sinusoidal motion has been built and experimentally tested. With the tools that iTaSC provides, along with the possibility to exploit the back-drivability of robots, complex applications in which teleoperation is sided with sharedcontrol can be achieved, by exploiting task constraints and estimation techniques.

\section{REFERENCES}

Borghesan, G., Willaert, B., and De Schutter, J. (2012). A constraint-based programming approach to physical human-robot interaction. In Int. Conf. Robotics and Automation. Minnesota, USA.

De Schutter, J., De Laet, T., Rutgeerts, J., Decré, W., Smits, R., Aertbeliën, E., Claes, K., and Bruyninckx, H. (2007). Constraint-based task specification and estimation for sensor-based robot systems in the presence of geometric uncertainty. Int. J. Robotics Research, 26(5), 433-455.

Kobayashi, Y., Hoshi, T., Kawamura, K., and Fujie, M. (2007). Control method for surgical robot to prevent overload at vulnerable tissue. In Int. Conf. Robotics and Automation, 1893-1899. Rome, Italy.

Kragic, D., Marayong, P., Li, M., Okamura, A.M., and Hager, G.D. (2005). Human-machine collaborative systems for microsurgical applications. Int. J. Robotics Research, 24(9), 731-741.

Lefebvre, T., Bruyninckx, H., and De Schutter, J. (2003). Polyhedral contact formation modeling and identification for autonomous compliant motion. IEEE Trans. Rob. Automation, 19(1), 26-41. 


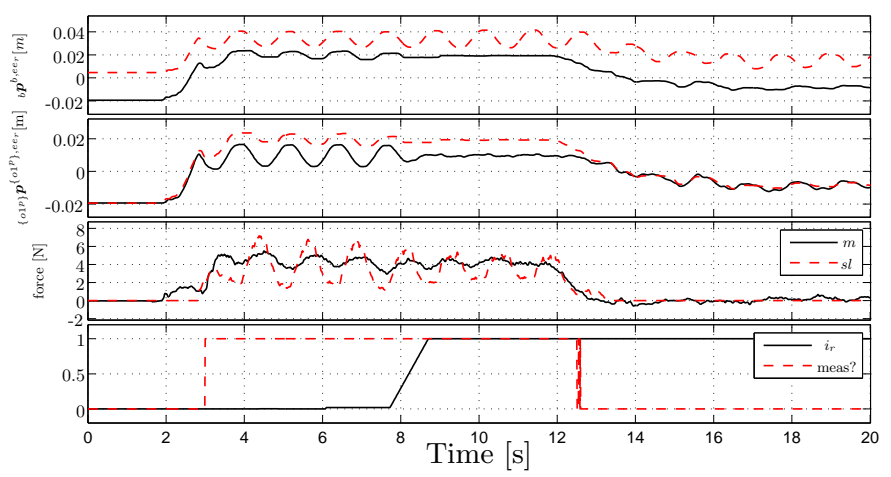

(a) position-position

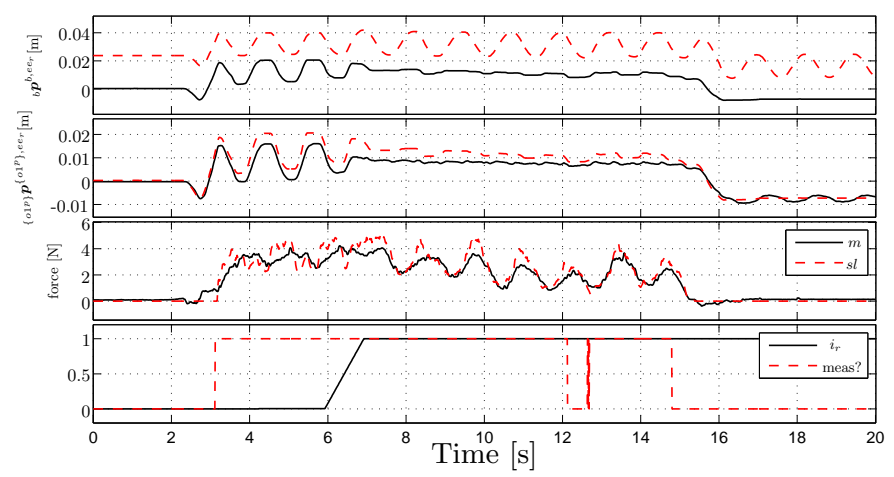

(b) position-force

Fig. 5. Position of real master and slave end-effectors, w.r.t their base ${ }_{b} \boldsymbol{p}^{b, e e_{r}}$ (first row), w.r.t. $\left\{o 1^{p}\right\}\left\{o 1^{p}\right\} \boldsymbol{p}^{\left\{01^{p}\right\}, e e_{r}}$ (second row), measured forces (third row), confidence index $i_{r}$ and contact (true/false) (fourth row).

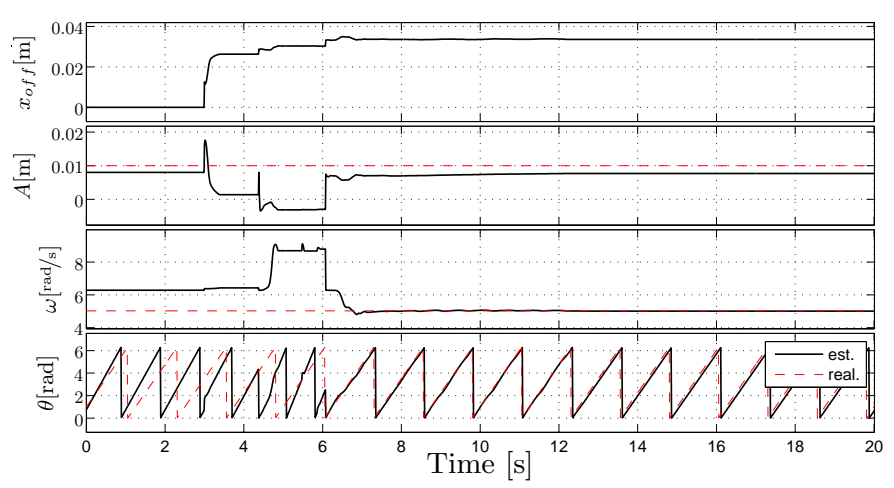

(a) position-position

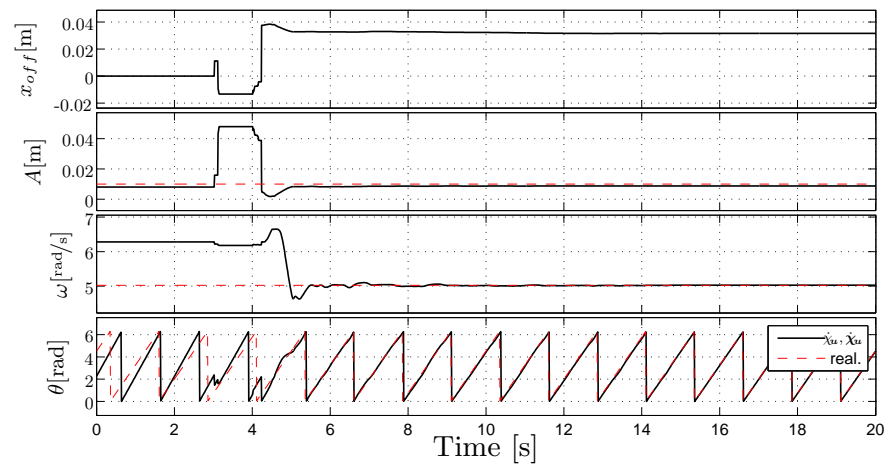

(b) position-force

Fig. 6. Estimated state $\boldsymbol{x}=\left[x_{o f f}, A, \omega, \theta\right]$ of the Extended Kalman filter, along with true values.

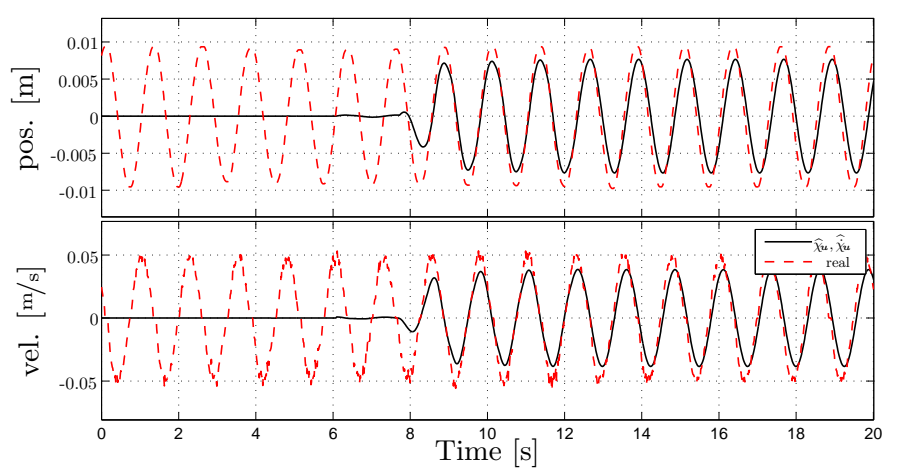

(a) position-position

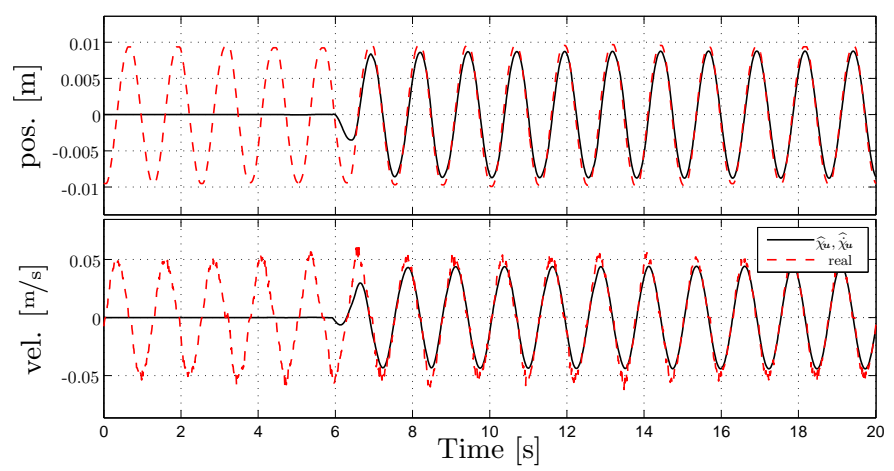

(b) position-force

Fig. 7. Real and estimated positions and velocities of the heart.

Massie, T.H. and Salisbury, J.K. (1994). The phantom haptic interface: A device for probing virtual objects. In Proceedings of the ASME Winter Annual Meeting, Symposium on Haptic Interfaces for Virtual Environment and Teleoperator Systems.

Ren, J., Patel, R.V., McIsaac, K.a., Guiraudon, G., and Peters, T.M. (2008). Dynamic 3-d virtual fixtures for minimally invasive beating heart procedures. IEEE Trans. Medical Imaging, 27(8), 1061-70.

Vanthienen, D., De Laet, T., Smits, R., and Bruyninckx, H. (2011). itasc software. http://www.orocos.org/itasc. Last visited January 2012

Willaert, B., Corteville, B., Vlem, J.V., Vanwynsberghe, K., Reynaerts, D., and Van Brussel, H. (2012). A multipurpose haptic device for research on physical humanrobot interaction. In Int. Conf. on New Actuators (in press).

Yuen, S.G., Kettler, D.T., Novotny, P.M., Plowes, R.D., and Howe, R.D. (2009). Robotic motion compensation for beating heart intracardiac surgery. Int. J. Robotics Research, 28(10), 1355-1372. 